\title{
Gating: Keep It Regular
}

Thomas Morneau, CNMT RT(N)

\section{INTRODUCTION}

Gating of SPECT myocardial perfusion imaging (MPI) to assess wall motion and left ventricular ejection fraction (LVEF) was first performed in the 1980s. Before that time, only planar, and later SPECT, MPI was interpreted and reported. One disadvantage of perfusion-only interpretations was the inability to distinguish between a true fixed defect and an artifact. A mild to moderate perfusion defect in areas of overlying soft-tissue deposition could be due to either attenuation or a prior myocardial infarction. In addition, it was also unclear whether these defects were due to a prior myocardial infarction, severe ischemia, or a combination of disease processes.

With the development of gated SPECT MPI, many of these uncertainties became reasonably clarified by the ability to evaluate left ventricular contraction (function) along with myocardial perfusion. This innovation in cardiac imaging revolutionized the field, and SPECT MPI became the standard test for evaluation of left ventricular perfusion and function.

During a SPECT myocardial acquisition, the $\gamma$-camera records photons at multiple projection angles, usually in a $180^{\circ}$ arc. A static image is acquired at each stop (60-64 projections) during the acquisition. These images are summed after acquisition to create SPECT tomographic images, traditionally displayed in 3 imaging planes (coronal, sagittal, and transverse) for image interpretation.

During gated SPECT MPI, the acquisition begins with the $\mathrm{R}$ wave on the patient's electrocardiograph, which corresponds to end-diastole. One cardiac cycle is divided into multiple frames or bins (8 or 16 depending on the system) of equal duration. Image data for each frame are acquired and stored separately throughout the acquisition.

During processing, the data from each frame or bin are summed. Storage in each bin is as follows: bin $1=$ enddiastole, bin $4=$ end-systole, and bin $8=$ end-diastole. The count statistics of these summed images should be identical to those of the nongated images.

Historically, 8 frames per cardiac cycle have been considered satisfactory. Acquiring images in this manner does not significantly change the acquisition time, and sufficient counts can be achieved even with the lower count statistics obtained for the lower ${ }^{99 \mathrm{~m}} \mathrm{Tc}$-labeled radiopharmaceutical doses (185-370 MBq [5-10 mCi]) routinely used today. Using 16-frame acquisitions provides better resolution; however, this type of acquisition results in a shorter duration for each frame.

COPYRIGHT @ 2020 by the Society of Nuclear Medicine and Molecular Imaging.
Consequently, if the acquisition time is not increased, insufficient data will be obtained, creating inferior and unreliable results. A 16-frame acquisition may also result in slightly higher LVEF calculations requiring normal limits to be adjusted.

Each frame (bin) in the acquisition must have a sufficient count density to produce a quality gated SPECT MPI study. Insufficient counts in any bin may produce a flashing or streak artifact, which may produce a negative effect on MPI as well as an inconclusive result.

\section{COMMON ISSUES AND RECOMMENDATIONS IN GATED ACQUISITION}

\section{Variations in Heart Rate}

When performing gated SPECT MPI, technologists should be aware that most patients rarely present with a completely regular heart rate during the acquisition. This issue is particularly common during postexercise imaging. An irregular heart rate can lead to various artifacts causing difficulty in interpreting the images.

- Beat Rejection Software: All current computer systems offer beat rejection software and the ability to set a range of acceptable beats per window (usually as a percentage) for acquisition. This window is based on the patient's R-to-R interval. The window setting (usually from $10 \%$ to $100 \%$ ) represents the variation in heart rate deemed to be acceptable. For example, the duration of a heartbeat in a patient with a heart rate of $72 \mathrm{bpm}$ is $0.8 \mathrm{~s}$. Therefore, a $20 \%$ window for gated acquisition would result in acceptance of all beats between 0.72 and $0.88 \mathrm{~s}$. Any beats outside this range will be rejected. As the window percentage increases, a greater number of irregular beats are accepted within the R-to-R interval, negatively affecting the accuracy of the LVEF when images are processed.

- Heart Rhythm Assessment: Patients with sinus rhythm are ideal candidates for gated SPECT MPI. Patients with mild tachy- or bradyarrhythmias are also acceptable candidates. However, severe arrhythmias (atrial fibrillation, frequent premature ventricular contractions, or certain types of heart block) result in a poor-quality gated acquisition that may affect the accuracy of LVEF and observed wall motion abnormalities.

- Data Storage: Fortunately, many SPECT systems are able to store the gated and raw perfusion data separately. This separate storage is helpful when issues arise with the gated data and prevents jeopardizing the perfusion data collected. 


\section{Gated Acquisition Setup}

To obtain the best possible gated information, it is imperative that technologists attempt to obtain the most reliable $\mathrm{R}$-to-R interval data possible.

- Lead Attachment: In prepping the patient for image gating, the technologist must ensure that the leads are securely attached. Although the medical professional who preps the patient for the stress test usually performs an excellent job of attaching the leads, the stress test itself (particularly if it is exercise stress) can affect the attachment.

- Lead Placement: Gated acquisition requires 3 leads: left arm, right arm, and left leg. The arm leads are placed on the upper chest, and the leg lead is placed on the left lower rib area. If this placement is suboptimal, it may be helpful to place the arm lead on the patient's inner wrist, similarly to the placement for a standard 12-lead electrocardiograph. The goal is to optimize placement so that the electrocardiograph tracing can track similarly to a standard 12-lead electrocardiograph, with a pronounced (higher-voltage) monophasic or reversed S-wave QRS complex and a relatively lower-voltage $\mathrm{T}$ and $\mathrm{P}$ wave (Fig. 1).

- System Detection of Cardiac Cycle: The goal is to ensure that the system is tracing the R-wave or reversed S-wave QRS complex, because this interval is what must be measured to provide an accurate LVEF for interpretation. Patient body habitus will sometimes affect where leads should be placed to acquire the ideal electrocardiograph pattern. The gating equipment supplied with different camera systems usually provides several lead configurations and voltage adjustments to assist in finding the optimal QRS complex, allowing the system to properly gate the study. If it is difficult to find an acceptable R-to-R interval on a particular patient's electrocardiograph, the technologist might have to use other standard areas for lead placement. Trying multiple or different lead locations is usually a good start (e.g., putting the upper leads on the patient's arms or inner wrists). If gating parameters cannot be changed on the system, one can ask the manufacturer for help in fine-tuning the acquisition set-up.

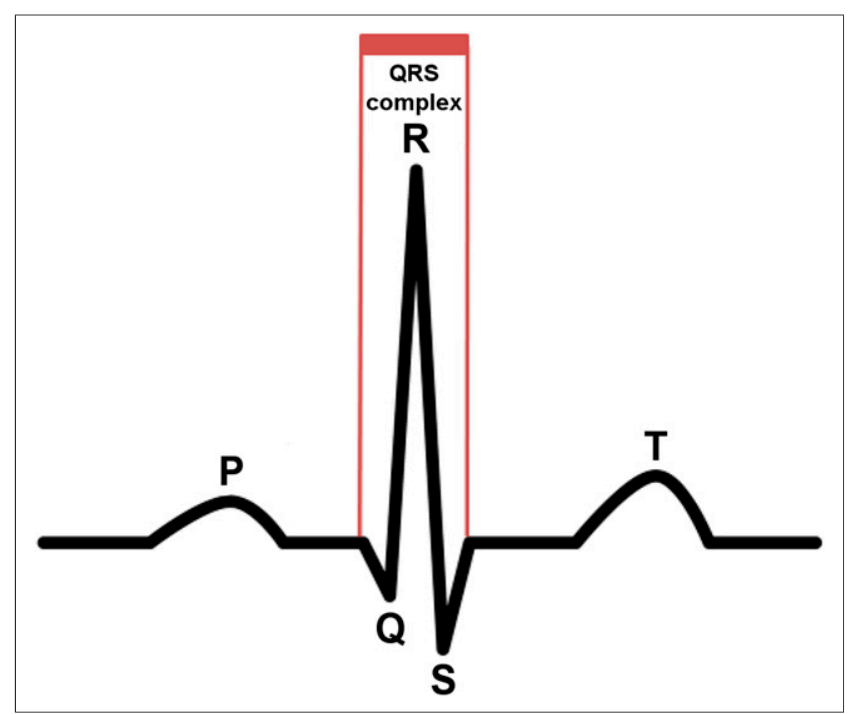

FIGURE 1. Gating window set to optimize and track QRS complex.

- Failed Gating: Under certain circumstances—such as in a patient who has arrhythmias during the studygated images cannot be acquired. In these cases, it is important to tell the interpreting physician about all the correction techniques that were attempted for gating. Most physicians who routinely interpret MPI studies are aware of the challenges that inaccurate data present and that such data will only compromise the results.

\section{REFERENCES}

1. Corbett JR, Akinboboye OO, Bacharach SL, et al. Equilibrium radionuclide angiocardiography. J Nucl Cardiol. 2006;13:e56-e79.

2. Paul AK, Nabi HA. Gated myocardial perfusion SPECT: basic principles, technical aspects, and clinical applications. J Nucl Med Technol. 2004;32: 179-187.

3. Scheiner J, Sinusas A, Wittry MD, et al. Society of Nuclear Medicine procedure guideline for gated equilibrium radionuclide ventriculography: version 3.0. In: Society of Nuclear Medicine Procedure Guidelines Manual. Reston, VA: Society of Nuclear Medicine and Molecular Imaging; 2002.

4. Zaret B, Beller G. Clinical Nuclear Cardiology. Philadelphia, PA: Mosby/ Elsevier; 2010:758-761. 